\title{
Modelling the criticality of silicon nitride surface imperfections under rolling and sliding contact
}

\author{
V. Zolotarevskiy ${ }^{a, *, 1}$, Y. Kadin ${ }^{b}$, C. Vieillard ${ }^{b}$ and M. Hadfield ${ }^{a}$ \\ ${ }^{a}$ Department of Design \& Engineering, Bournemouth University - Poole, United Kingdom \\ ${ }^{b}$ SKF Research \& Technology Development - Houten, The Netherlands
}

\section{ARTICLE INFO}

\section{Keywords:}

Surface defects

Rolling Contact Fatigue

Hybrid Bearings

Ceramics

\begin{abstract}
A B S T R ACT
Ceramic rolling elements of hybrid bearings may initially include surface imperfections. In order to provide reliable operation of a bearing, the criticality of such imperfections under rolling contact fatigue is examined by defining them as Star features: intersecting semi-elliptical surface cracks. Parametric study is conducted using Finite Element Method and discussed with help of previously published experimental observations. The effects of the Star feature morphology and configuration, contact pressure and crack face friction are investigated in terms of stress intensity factors. Possible crack propagation scenarios are explained in the present study.
\end{abstract}

\section{Introduction}

Rolling contact fatigue (RCF) is a well-known type of failure in bearings. Local RCF damage manifested by crack initiation and propagation, can be related to one of two modes: subsurface or surface initiated fatigue. Subsurface fatigue is typically caused by material defects (non-metallic inclusions, porosity, etc.), which lead to stress concentration and initiation of fatigue cracks. The surface initiated fatigue is typically associated with poor lubrication conditions when the mating surfaces are not fully separated by the lubricant film and high stresses are produced on the asperity level. However, the surface initiated fatigue can also be applied to pre-existing material defects and imperfections, which may occur in silicon nitride $\left(\mathrm{Si}_{3} \mathrm{~N}_{4}\right)$ ceramic components of hybrid bearings. In many applications, these bearings overperform full-steel bearings (see $[1,2]$ ), and therefore the research in the field of hybrid bearings and $\mathrm{Si}_{3} \mathrm{~N}_{4}$ is of high interest.

Although $\mathrm{Si}_{3} \mathrm{~N}_{4}$ ceramics are superior over traditional bearing material in multiple aspects (lightness, high hardness, good resistance to corrosion, electrical insulation, etc.), it is still less resistant to crack initiation and propagation, compared to steels. Pre-existing material imperfections can be present on surfaces of ceramic rolling elements being the result of manufacturing (by sintering) and/or surface finishing processes. There exists a risk that these imperfections can trigger propagation of fatigue cracks during bearing operation, which eventually leads to material spalling from rolling surfaces.

One of the techniques to mimic surface defects in $\mathrm{Si}_{3} \mathrm{~N}_{4}$ ceramics is to use a sharp diamond (e.g. Vickers) indentation, which in non-ductile materials typically results in formation of cracks (see Fig. 1). These cracks have a similarity to specific types of imperfections which can be observed

*Corresponding author

v.zolotarevskiy@deakin.edu.au (V. Zolotarevskiy) $\operatorname{ORCID}(\mathrm{s})$ :

${ }^{1}$ Present address: Institute for Frontier Materials, Deakin University Waurn Ponds VIC, Australia.
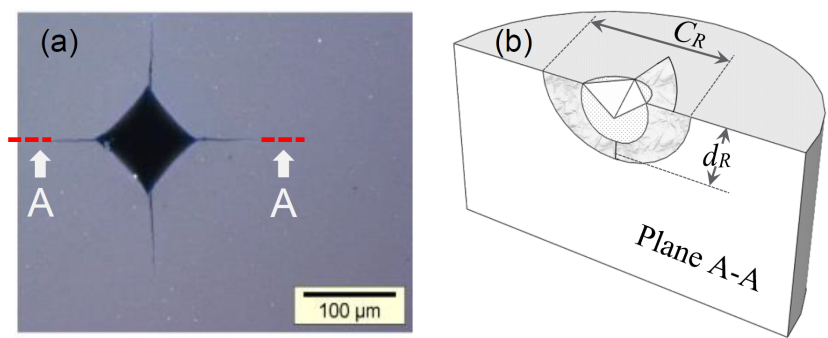

Figure 1: A Vickers indented surface of ceramics: (a) the indentation leads to the formation of radial cracks emanating from the indent. 3D schematic of the Vickers cracks morphology (b).

on surfaces of $\mathrm{Si}_{3} \mathrm{~N}_{4}$ rolling elements. After the indentation, artificially damaged rolling elements are investigated in an RCF test for the inspection of crack propagation and development of spalls. This experimental technique is quite efficient, because it allows the creation features that are a sufficiently large size and of repeatable geometry, which enables to run an RCF experiment under controlled conditions. Another benefit of this technique is the Vickers indentation test is used for the toughness examination in brittle materials which requires measurement of surface crack and modelling of its formation (see e.g. [3]). Due to this knowledge the size of the artificially created crack and its shape can be theoretically predicted [4]. The Vickers indented $\mathrm{Si}_{3} \mathrm{~N}_{4}$ balls were run in the four-ball machine test which showed that the main failure associated with the artificial cracks was surface delamination and spalling (see Refs. [5, 6]). In [7] and [8] the RCF tests were carried out by a thrust-type machine using the $\mathrm{Si}_{3} \mathrm{~N}_{4}$ Vickers indented plate. The fatigue propagation of surface cracks and their branching from an initial crack were observed, and the process of flaking failure was investigated. The Vickers indented (prismatic) $\mathrm{Si}_{3} \mathrm{~N}_{4}$ samples were tested in the torsion-bending and in the reciprocating RCF machine [9] investigating the friction effect on surface cracks growth.

Analyzing surface cracks in $\mathrm{Si}_{3} \mathrm{~N}_{4}$, the authors of [7] and [9] used fracture mechanics in order to explain the mode of 
crack propagation observed in the experiments. It is not unusual to use fracture mechanics in the modelling of RCF in both metallic and ceramic materials. The authors' previous studies used fracture mechanics for the modelling of other types of surface damages appearing in $\mathrm{Si}_{3} \mathrm{~N}_{4}$ rolling elements of hybrid bearings: linear elastic fracture mechanics was used in [10] for the modelling of the so-called C-crack and the concept of the generalized stress intensity factor was used in [11] for the modelling of missing material. The surface cracks obtained in the Vickers indentation were simplistically modeled in previous studies [7] and [9] as semielliptical cracks, excluding the systematic study intending to relate the dimensions of Vickers cracks and contact conditions to the risk of their fatigue propagation. In the present study a surface damage termed "Star Feature" is introduced (see e.g. Ref. [12]). Such damage is represented by a group of cracks, which has at least one common location of cracks intersection. These locations may be in some cases associated with a pit due to material removal, or by existence of plastic zone. A typical image of a Star-shaped damage on a ceramic surface is shown in Fig. 2. The shape of this naturally formed feature, has some similarity with the artificially formed Vickers indentation. The morphology depicted in Fig. 2 also consists of radial cracks (perpendicular to raceway) crossing each other. So, mimicking Star feature artificially in terms of Vickers indentation by creating intersecting radial cracks is relevant to the analysis of natural Star-like damage. In order to explore this type of damage, it is wise to start with the most trivial case of Star feature - a crossintersection of two similar cracks (see Fig. 1).

Sintered ceramics are heterogeneous materials, because they consist of more than one phase (see e.g. [13, 14]) and the grains of $\mathrm{Si}_{3} \mathrm{~N}_{4}$ are anisotropic [15]. However, due to massive amount of grains and their random orientation, such polycrystalline materials as sintered $\mathrm{Si}_{3} \mathrm{~N}_{4}$ can be considered as continuum media, and their mechanical properties can be homogenized, including the properties responsible for cracking [14].

The current work develops a Finite Elements model for the simulation of surface cracks formed by the Vickers indentation and cyclically loaded afterwards, via rolling or sliding contact. This model is based on fracture mechanics and aims to compute Stress Intensity Factor (SIF) (see Ref. [16]), which can be related to the fatigue growth behavior of surface cracks. An intensive parametric study is run in order to relate the geometry of Vickers cracks and the rolling/sliding contact conditions to SIF and by this to assess the risk of fatigue failure. It is obvious that the artificially created imperfection (see Fig. 1) has much more controlled geometry than a naturally formed Star in Fig. 2. Due to the fact that it very difficult (if ever possible) to determine a most characteristic shape of a natural Star, an "idealized" Star morphology, correlated well with the artificially produced feature was introduced for the current modelling. This "idealized" morphology is described by the number of geometrical parameters, and by varying these parameters in FE model the criticality of Star can be assessed. Within admit in this current work, the damage can vary in its orientation relative to the overolling direction, and can be shifted relative to the contact zone centre, and have different geometries. The goal of the current work is to investigate the effect of these parameters on the criticality of crack propagation, which according to fracture mechanics can be quantified in terms of SIF.

It is important to note, that the current study assumes a pre-existing crack of given geometry while in future, more advanced study combining XFEM (or adaptive re-meshing technique) with cohesive elements model, can be conducted. In such a study the assumption of the pre-existing crack can be relaxed, by simulating explicitly the crack initiation and its propagation in RCF. The XFEM based simulation of crack propagation under RCF in rails was presented in [17], and simulation of crack initiation/propagation under uniaxial load in $\mathrm{Si}_{3} \mathrm{~N}_{4}$ using a cohesive elements model with adaptive remeshing in [14]. Note, that differently from [14], where only the mode I (crack opening) was considered, the RCF simulations have to relate to the mixed mode and to the crack faces contact interaction (occurring when a crack is closed under compressive stress). It is fraught with high level complexities in mathematical formulation and numerical implementation leading to high computational effort (time consuming calculations) and possibly to convergence problem.

\section{Description of the model}

In general, RCF is a multi-axial fatigue in which subsurface crack propagation is driven by the mixed mode, including the modes I, II and III. The modes II and III are driven by shear stresses, while the mode I by the tensile stress, locally appearing at the edge of the point contact and is enhanced due to the transition to sliding conditions (both conditions are considered in the current work). However, these tensile stresses are confined in small zones, thus a radial crack of relatively large size can be predominantly subjected to compressive stress (which as is known, prevails in contact). As a result, the faces of the crack enter into contact with each other, which suppresses the opening mode I and leads to frictional interaction between faces of the crack. Crack faces are typically rough and the friction between them is characterized here by the friction coefficient, which is generally to establish unknown, because an experimental measurement of this parameter is difficult if ever possible (see Ref. [18]). Therefore, it is not fixed but is included into the parametric study along with other variables.

In order to distinguish the full film lubrication conditions from the poor lubrication (thin film), we introduce in a model with two different conditions: rolling (corresponding to full film) and sliding (thin film). It should be mentioned, however, that in reality bearings can run under different lubrication conditions, which is expressed by the lambda ratio (see e.g. Ref. [20]).

Figure 3 schematically presents the contact between a ball and a substrate. For simulation of a rolling contact problem, a small angle is initially created between the top surface of the half ball and the rolling direction - such an angle van- 


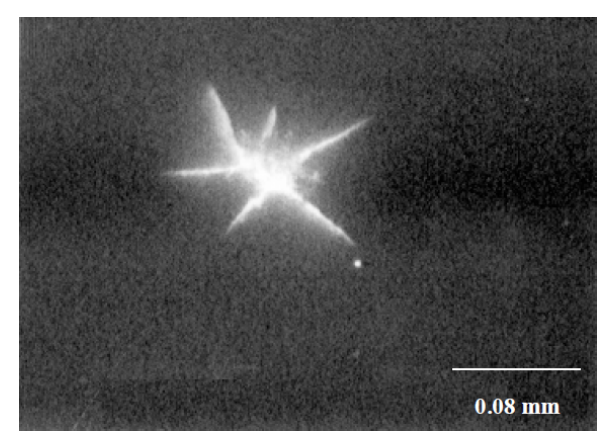

Figure 2: An example of a natural Star feature found on ceramic surfaces under UV light (see Ref. [19].)

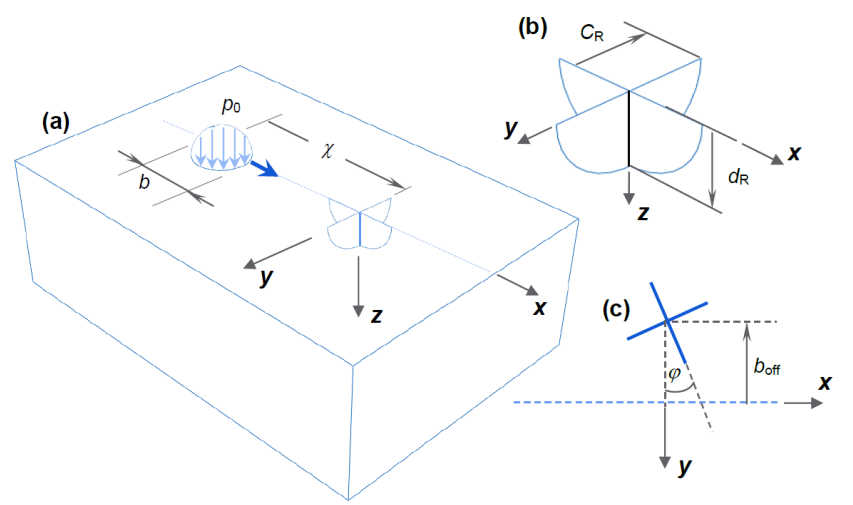

Figure 3: Schematic of the contact problem in presence of a Star feature.

ishes in sliding and rotation is restricted because the sliding body does not change its orientation during motion. In this work a contact is produced in terms of a point contact (contact of circular shape - see [21]). Such a contact (differently from the cylindrical equivalent to the plane strain contact) leads to the formation of local tension on the surface (this tension vanishes as the elliptical contact gets sufficiently long coinciding with the plain strain conditions), and consequently activates the $\bar{K}_{I}$ mode. This mode is present according to our model but can be suppressed in the case of a real bearing, where the contact zone is elliptical which leads to quite lower tension on a raceway. The reason for using the point contact and not the elliptical contact (which in reality takes place in a bearing) is for the sake of comparison to experimental studies of RCF in the presence of surface Star features. Such experiments are conducted in special testing apparatuses, like ball rolling on an artificially featured (indented) plate (see e.g. a 6-points rolling contact tests in Ref. [7] and a 4-ball tests in Ref. [22]. The contact zone in all these apparatuses is indeed circular, which explains the choice to implement a point contact in the numerical model for the present study.

Figure 4 illustrates a Star feature as a perpendicular intersection of semi-elliptical surface cracks embedded in the substrate, when either friction or frictionless contact conditions may be specified at the crack faces. It is important to note, that the Star model developed in the current work ex- cludes existence of a plastic zone, which is formed along with radial cracks during the Vickers indentation [4]. This plastic zone can induce some residual tension in the vicinity of Star, which can reduce the crack faces friction (which, strictly speaking, is unknown, but is studied parametrically in the current work). Experimental measurement of this localised residual stress is very challenging, because it occupies very small material volume. Hence, for the sake of simplicity this effect is omitted from the current study. The geometry of the elliptical cracks is defined by the radial crack half width $C_{R}$ and radial crack depth $d_{R}$. Here $0^{\circ} \leq \theta \leq$ $180^{\circ}$ is an angle that defines the position along the crack front, and points $A, B$ and $B^{*}$ represent the deepest and the surface locations of the crack front, respectively (see Fig. 4(a)). It is known, that for accurate computation of SIFs a crack front has to be properly discretized, which obviously leads to high amount of elements in the model and increases computational time. Therefore, the model efficiency (in terms of computational time) can be improved by using the symmetry of the considered feature. For this, only one crack of the Star is finely meshed along the front, while another half of the feature is present only to model properly, the full morphology of the Star. Here, the finely and the roughly meshed cracks are termed as a "primary" crack (PC) and a "secondary" crack (SC), respectively (see Fig. 4(b)). Obviously, the computation of SIFs is performed only for the primary crack, whose orientation is relative to the rolling direction and its effect on crack propagation is parametrically studied in the current work by the rotation of the substrate containing the Star feature in terms of an angle $\varphi$, see Fig. 3.

The problem was solved numerically by the Finite Element Method (FEM) using a commercial package ABAQUS. The three-dimensional FE mesh was consisted of linear hexahedral elements (C3D8R). The sphere and the substrate were divided into different mesh density zones, with the highest mesh density concentrated at the crack front. The FE model and the meshed embedded cracks are presented in Fig. 5.

The total number of elements in the numerical model is $\approx 53,000$, however, a detailed analysis on the mesh density will be addressed later.

In order to calculate SIFs, the crack opening/sliding displacement method is used (see e.g. Ref. [23]):

$$
\begin{aligned}
K_{I} & =\frac{E}{4\left(1-v^{2}\right)} \sqrt{\frac{2 \pi}{r}}\left(u_{1}-u_{2}\right), \\
K_{I I} & =\frac{E}{4\left(1-v^{2}\right)} \sqrt{\frac{2 \pi}{r}}\left(v_{1}-v_{2}\right), \\
K_{I I I} & =\frac{E}{4(1+v)} \sqrt{\frac{2 \pi}{r}}\left(w_{1}-w_{2}\right),
\end{aligned}
$$

where $u, v, w$ are displacements corresponding to the directions $x, y, z$ respectively, the indexes 1,2 relate to opposite crack faces, $r$ is the distance from the crack tip and $E, v$ are Young's modulus and Poisson's ratio respectively. A script in the ABAQUS programming environment Python was written for the sake of such calculations. Note, that the 

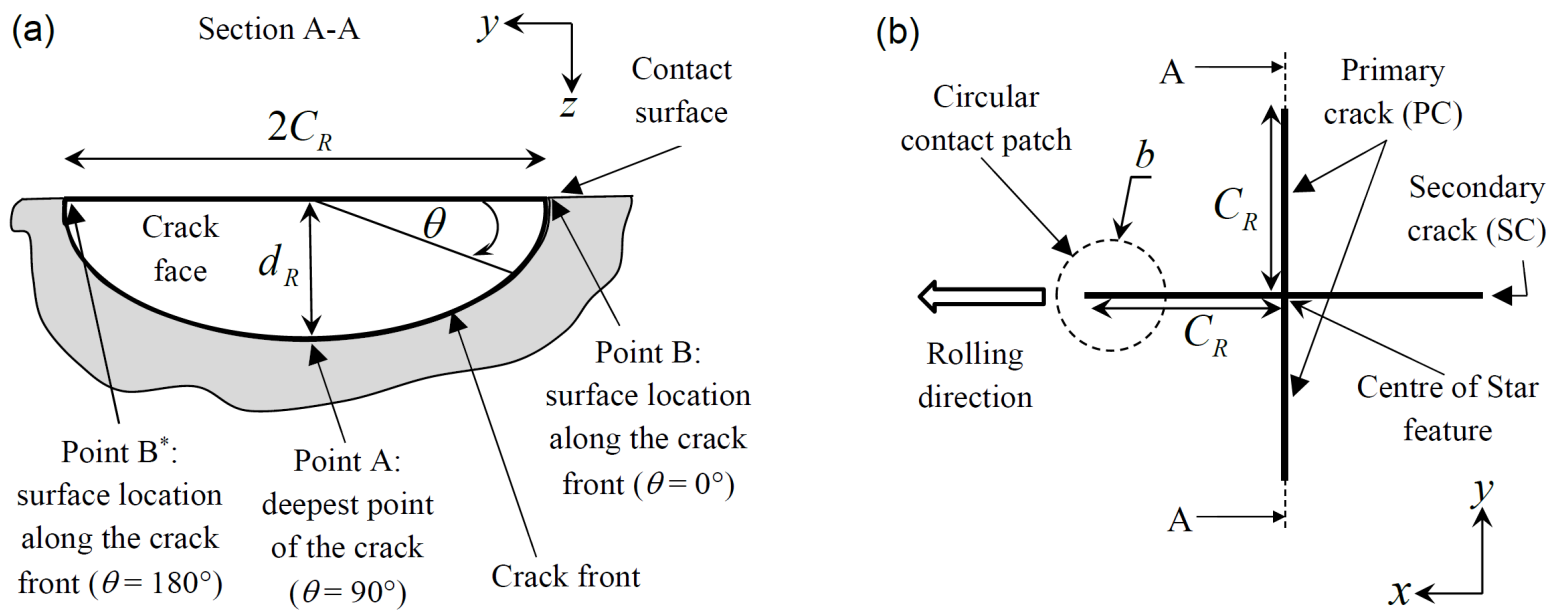

Figure 4: Sketch of (a) cross section and (b) top view of the Star feature.

Table 1

Dimensionless simulation parameters.

\begin{tabular}{ll}
\hline Parameter & Simulation values \\
\hline$C_{R} / b$ & $0.05,0.2,0.5,0.75,1,2$ \\
$d_{R} / C_{R}$ & $0.3,0.5,0.8,1$ \\
$\mu_{\text {crack }}$ & $0,0.3,0.7,1$ \\
$\mu_{\text {surface }}$ & $0.05,0.1,0.15$ \\
\hline
\end{tabular}

crack front was meshed with the same element type as everywhere in the model: no special singular element (implemented sometimes for crack problems) was used.

The parametric study was run for the parameters in Tab. 1 , defining the geometry of the Star and friction conditions. Here $\mu_{\text {crack }}$ and $\mu_{\text {surface }}$ are the friction coefficients at the crack face and on the contact surface of the ball and the substrate respectively. The material properties are defined by Young's modulus ( $E=305 \mathrm{GPa}$ ) and Poisson's ratio $(v=0.27)$ for all the components of the model. The latter parameters were chosen to match the properties of $\mathrm{Si}_{3} \mathrm{~N}_{4}$ (see Ref. [24]).

\section{Model setup}

The rolling and sliding contact problem that was simulated in this study is presented in Fig. 6. Ball radius $R_{w}$ and normal load $P$ were calculated according to Hertz solution for a given pressure $p_{0}$, termed as the maximum Hertzian contact pressure (see Ref. [21]). The normal load $P$ is applied at the symmetry plane of the ball in $-z$ direction, which produces a contact radius $b$ (see Figs. 3 and 6). This load is maintained as the ball motion (rolling or sliding) is established by applying the horizontal displacement in $x$ direction to simulate a contact problem in the presence of surface Star feature (see Fig. 6) embedded in the fixed substrate which side faces and the bottom are clamped.

The centre of the Star feature was placed at the origin of the global coordinate system and the ball was initially located at a specified distance from the crack centre, described by the position on the $x$ axis. This position is defined by:

$$
\chi_{\max }=\frac{3}{2} b+C_{R}
$$

where $\chi$ stands for a rolling path (see Fig. 3(a)). The relation 2 ideally covers the path required to obtain the expected stress field, as well as minima and maxima of the SIFs for the present parametric study. So, the total overolling distance was $2 \chi_{\max }$ (i.e. the entire interval $\chi \in\left[-\chi_{\max }, \chi_{\max }\right]$ ), by equally divided loading increment steps. For the simulation of the rolling conditions, the ball was initially inclined with respect to the $x$ axis by a small angle calculated in terms of the relation $\gamma=\arctan \left(\chi_{\max } / R_{w}\right)$ (see Fig. 6(a)) for $\chi_{\max }$ in Eq. 2. The angle $\gamma$ was adopted to establish proper contact between ball and substrate and to eliminate convergence and stability issues. In other words, while the ball rotates due to the prescribed displacement, such an angle guarantees that the fine mesh of the ball matches the fine mesh of the substrate. For sliding contact conditions $\gamma=0^{\circ}$ (see Fig. 6(b)) because the ball rotation is excluded for this contact condition. SIFs were calculated on the semi-elliptical crack front for each loading increment. Because the study relates to fatigue, it is convenient to represent the SIF results in terms of the expression

$$
\Delta K \equiv K_{\max }-K_{\min },
$$

where we define $K_{\max }$ and $K_{\min }$ as the maximum and the minimum values of SIFs determined from the result set. $K_{\max }$ and $K_{\min }$ were obtained for a given $\theta$. In this work the SIF are presented in terms of a dimensionless geometric factor $\bar{K}$, which is determined by the crack shape and the contact pressure:

$$
\bar{K}_{I}=\frac{K_{I}}{p_{0} \sqrt{\pi C_{R}}} ; \quad \bar{K}_{I I}=\frac{K_{I I}}{p_{0} \sqrt{\pi d_{R}}} ; \bar{K}_{I I I}=\frac{K_{I I I}}{p_{0} \sqrt{\pi C_{R}}} .
$$



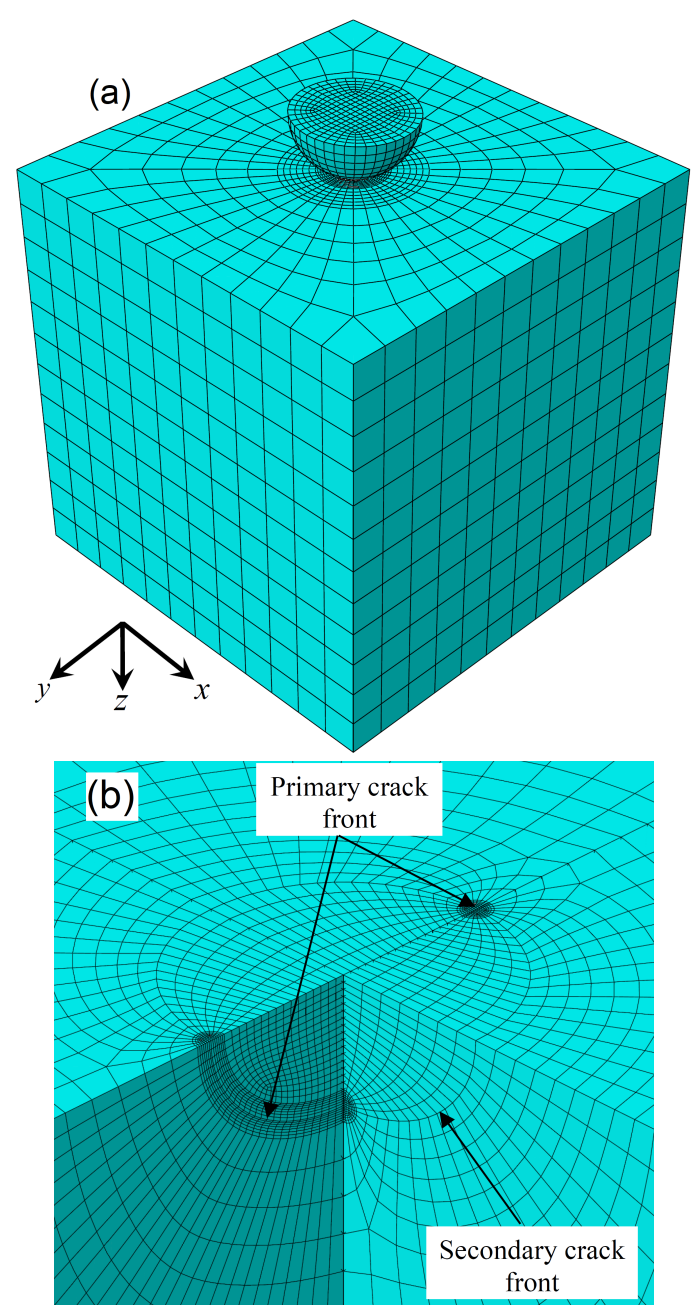

Figure 5: An isometric view of the full Finite element model (a) and an embedded Star feature (b) with one quarter of the substrate as well as the ball hidden. The fine mesh corresponds to the Primary crack front.

\section{Results and discussion}

\subsection{Validation and mesh analysis of the FE model}

The numerical model was verified with existing analytical and numerical solutions. In order to test the methodology for SIFs computation a simpler problem of a pennycrack was considered. The numerically computed SIFs were compared against the analytical solution for a circular penny crack embedded in infinite space and subjected to the tensile and shear stress (see Ref. [25]). This comparison is presented in Fig. 7 indicating the difference between the numerical and the analytical solution not higher than $4 \%$. Although the penny crack problem is not relevant for the modelling of Star, the current numerical validation provides us an important information regarding the mesh density at the crack front and the proper utilization of the crack opening/sliding displacement method. In addition, the present FE model was compared with the solution of the semi-elliptical surface crack in tension problem. This problem was solved using FEM (see Ref. [26]). For the sake of validation, the
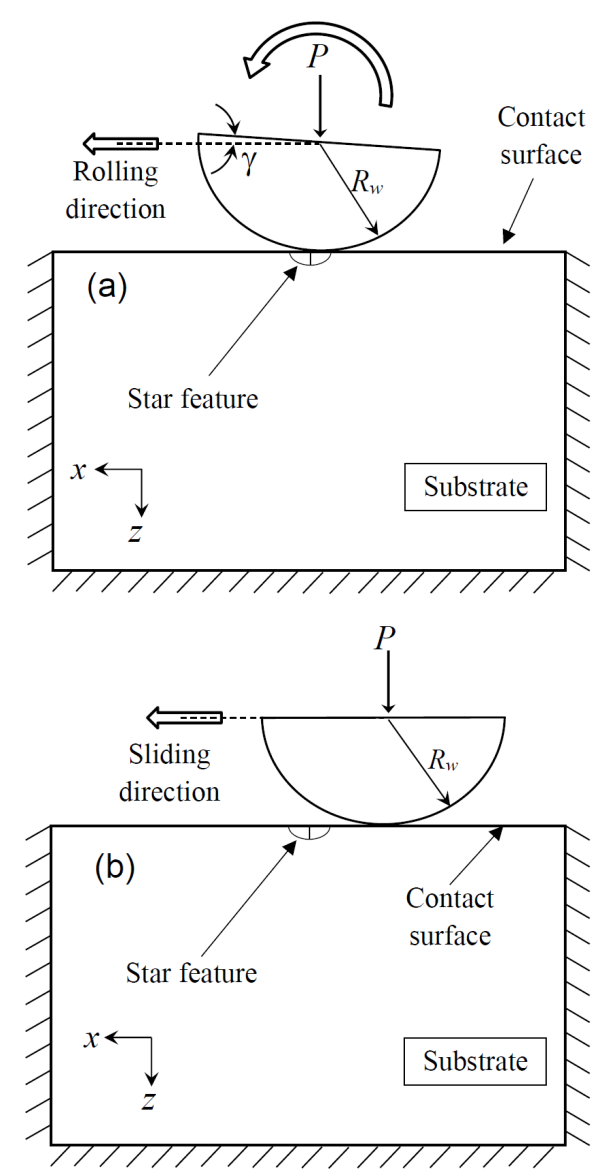

Figure 6: Schematic representation of rolling (a) and sliding (b) contact problem.

FE model of the present work was modified to match the boundary and loading conditions in Ref. [26] and set of tensile stresses $\sigma=1,10,100,200 \mathrm{MPa}$ was applied in normal direction to the crack face. For such conditions the geometric factor obtained in [26] is $\bar{K}_{I}=K_{I} /(\sigma \sqrt{\pi a})=0.7309$ ( $a$ is the crack size). In the present FE model for the set of $\sigma$ the geometric factor varied between 0.7420 and 0.7422 , so the difference in the SIF values with respect to the solution in Ref. [26] was within $1.5 \%$. The final validation of the FE model was with the Hertz contact problem. This analysis provided $1.9 \%$ relative error of the maximum contact pressure and $2.8 \%$ relative error of the contact radius. So, the results of the present numerical model provides good approximations in terms of contact and fracture mechanics modelling.

In order to run accurate yet time efficient simulations, the appropriate mesh and number of overolling loading increments had to be determined. Coarse mesh may provide a fast run time but poor accuracy, while exceptionally fine mesh requires long run times and expensive computational resources. Similar considerations are related to an optimum choice of loading increment, that applies to the discretisation of an overroll by substeps. It is, therefore a single loading step that then summed up to the total number of incre- 


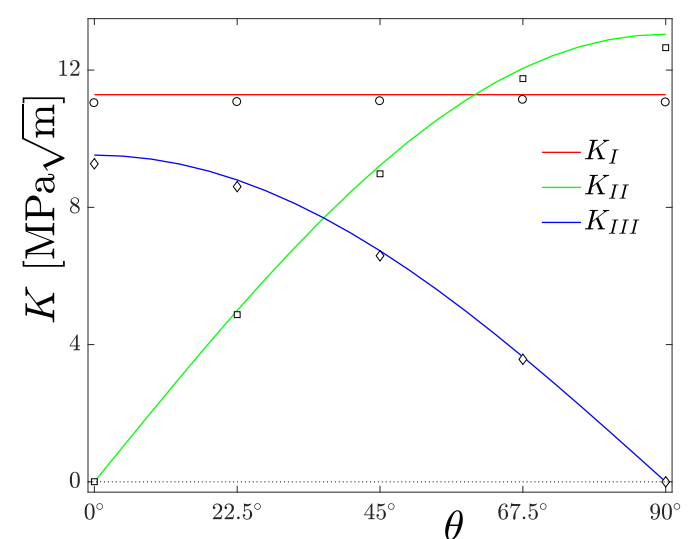

Figure 7: Distribution of SIFs along one quarter of the crack front $\left(0^{\circ} \leq \theta \leq 90^{\circ}\right)$ for validation of the FE model with a subsurface penny-crack embedded in an infinite space and loaded by tensile and shear stress. The FE results are presented with markers and the analytical solution with solid curves.

ments required to cover the entire distance $2 \chi_{\max }$ (see Fig. 3 ). So, the smaller the discrete loading intervals, the more intermediate SIF steps are obtained during the overolling needed to determine the extremal SIF values. As the number of such increments increases, the computing time also increases. Another important time factor is related to the postprocessing evaluation of the SIF, with more time needed to compute large number of loading increments.

For the sake of the mesh density and number of loading increment analysis, frictionless conditions are considered at the crack face and the rest of the parameters are described in Sec. 3. The reason for the choice $\mu_{\text {crack }}=0$ is due to the increased computing time for non-zero friction. In aims of the loading increment analysis, the set of 5, 10, 20, 50, 100 loading increments was investigated for a model with 53,324 elements in rolling contact problem. SIF were calculated using Eq. 3 along the crack front $\left(0^{\circ} \leq \theta \leq 180^{\circ}\right.$, see Fig. 4). Maximum values for each mode $\Delta K$ were determined and compared. The relative error for 20 loading increments with respect to 100 loading increments is $\{0.4 \%, 0.1 \%, 1.7 \%\}$ for $\left\{\max \Delta \bar{K}_{I}, \max \Delta \bar{K}_{I I}, \max \Delta \bar{K}_{I I I}\right\}$ respectively, so we adopted 20 loading increments during the rolling stage for the rest of the preliminary study.

The mesh density analysis was performed according to the following procedure: the simulation results were obtained from 6 models with different mesh density from $N_{1} \approx 32,000$ to $N_{6} \approx 361,000$ elements, and the convergence SIFs plots were produced - see Fig. 8. For each of the six models the finest mesh density region was created along the crack front, and the rest of the mesh regions were modelled to maintain a reasonable aspect ratio relative to the neighbouring regions as well as in the contact area. So, the relative errors in terms of the three SIF modes for the model $N_{3} \approx 53,000$ elements with respect to the highest mesh density model $N_{6} \approx$ 361,000 varied by less than $2.5 \%$. The model $N_{3}$ is time efficient - the run time using a single core with $\mu_{\text {crack }}=0$ is about 60 minutes, when the model $N_{4}$ runs about 100 min-
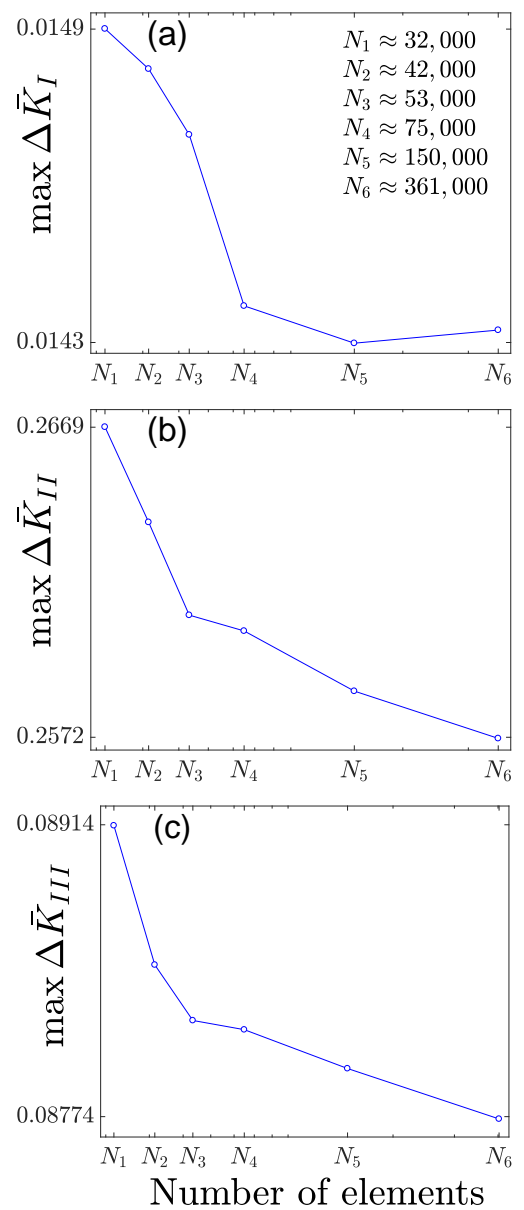

Figure 8: Maximum values of $\Delta \bar{K}_{I}$ (a), $\Delta \bar{K}_{I}$ (b) and $\Delta \bar{K}_{I I I}$ (c) along the crack front for models consisted of total number of elements $N_{1} \approx 32,000, N_{2} \approx 42,000, N_{3} \approx 53,000, N_{4} \approx$ $75,000, N_{5} \approx 148,000$ and $N_{6} \approx 361,000$ with $\mu_{\text {crack }}=0$, $C_{R} / b=0.75, d_{R} / C_{R}=0.8$ in rolling contact.

utes. In addition, the post-processing data is produced relatively fast by $N_{3}$, and according to our analysis it provides accurate results within a range of small numerical errors, so this model is adopted for the rest of the study.

\subsection{Analysis of SIFs for symmetrical configuration of the Star feature}

In terms of the evolution of SIF during overolling, for simplicity a representative Star scenario is considered as the most symmetric configuration with respect to the rolling orientation and the position of the contact patch relative to the Star. This configuration implies that the angle $\varphi$ (crack orientation relative to the motion of contact zone) is $0^{\circ}$ and the offset distance between the intersection of the cracks forming the Star and the rolling path is $b_{o f f}=0$ (see Fig. 3(c)). For such conditions only one crack - perpendicular to the direction of contact zone motion (primary crack) is loaded, while the second crack is free of any mode of crack propagation. This is because its plane lays on the symmetry plane, at which the shear stress vanishes and the crack experiences only compressive normal stress. In other words, for such a 

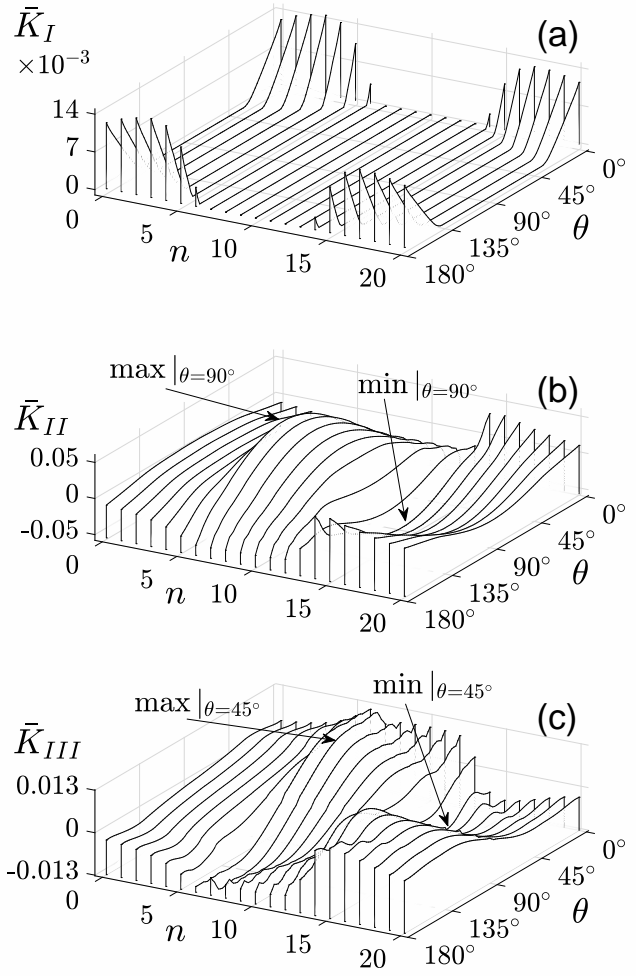

Figure 9: Distribution of $\bar{K}_{I}$ (a), $\bar{K}_{I I}$ (b) and $\bar{K}_{I I I}$ (c) along the crack front $\left(0^{\circ} \leq \theta \leq 180^{\circ}\right)$ having $\mu_{\text {crack }}=0.7$, $C_{R} / b=0.75, d_{R} / C_{R}=0.8$ for rolling contact conditions, with overolling path $\chi \in\left[-\chi_{\max }, \chi_{\max }\right]$ and loading increment number $n$. Here $\Delta \bar{K}_{I I}\left(90^{\circ}\right)=\bar{K}_{I I}^{(n=6)}-\bar{K}_{I I}^{(n=14)}$ at $\theta=90^{\circ}$ and $\Delta \bar{K}_{I I I}\left(45^{\circ}\right)=\bar{K}_{I I I}^{(n=7)}-\bar{K}_{I I I}^{(n=14)}$ at $\theta=45^{\circ}$.

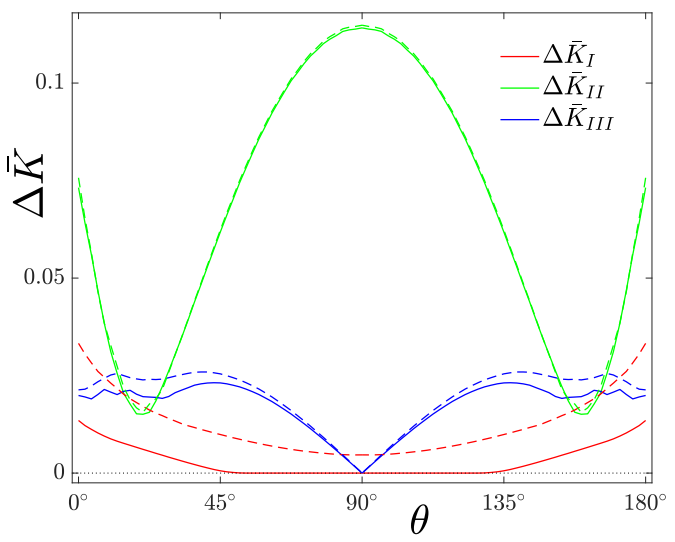

Figure 10: Distribution of $\Delta \bar{K}$ along the crack front $\left(0^{\circ} \leq\right.$ $\theta \leq 180^{\circ}$ ) for rolling (solid curves) and sliding (dashed curves) with $\mu_{\text {crack }}=0.7, C_{R} / b=0.75, d_{R} / C_{R}=0.8$.

symmetrical loading of the Star, the stress field of the primary crack is unaffected by the existence or absence of the secondary crack. Figure 9 shows a distribution of SIF $\bar{K}_{I}$, $\bar{K}_{I I}$ and $\bar{K}_{I I I}$ along the crack front $\left(0^{\circ} \leq \theta \leq 180^{\circ}\right.$, see Fig. 4) within rolling contact during overolling. The loading increment in Fig. 9 varies in terms of $n$, when the rolling of the half-ball was initialized and finalized at $\chi=\chi_{\max }(n=0)$ and $\chi=-\chi_{\max }(n=20)$, respectively. One can see that
$\bar{K}_{I}$ is mostly pronounced close to the surface $\left(\theta=0^{\circ}, 180^{\circ}\right)$ and vanishes at the crack front points located deeper in the substrate (see Fig. 9(a)). As it follows from Fig. 9(b), maximum and minimum values of $\bar{K}_{I I}$ are at the deepest crack front location, $\theta=90^{\circ}$ with $\bar{K}_{I I_{\max }}$ and $\bar{K}_{I I_{\min }}$ for $n=6$ and $n=14$ respectively. The extremal values of $\bar{K}_{I I I}$ were obtained at opposite locations of the crack front, $\theta=12^{\circ}$ and $\theta=168^{\circ}$ for $n=7$, but for fixed $\theta$ one can see in Fig. 9(c) that for example at $\theta=45^{\circ} \Delta \bar{K}_{I I I}=\bar{K}_{I I I}^{(n=7)}-\bar{K}_{I I I}^{(n=14)}$.

Distribution of $\Delta \bar{K}$ along the semi-elliptical crack front $\left(0^{\circ} \leq \theta \leq 180^{\circ}\right)$ for the rolling and sliding contact conditions (solid and dashed curves respectively) is illustrated in Fig. 10. We infer that $\Delta \bar{K}_{I}$ is lower than the modes $\Delta \bar{K}_{I I}$ and $\Delta \bar{K}_{I I I}$. The maximum value of $\Delta \bar{K}_{I I}$ and the minimum value of $\Delta \bar{K}_{I I I}$ are obtained at the deepest point of the crack $\left(\theta=90^{\circ}\right)$, and the distribution of all three modes is symmetrical. When rolling is restricted and pure sliding occurs, $\Delta \bar{K}_{I}$ is higher than 0 the for the entire crack front (see Fig. 10 ), and higher than $\Delta \bar{K}_{I}$ for rolling. The observed difference between rolling and sliding for the first mode will be discussed later. The second and the third modes are almost the same for both regimes.

The results presented in Figs. 9 and 10 indicate the complexity of the current problem related to the multi-axial nature of RCF. Indeed, a crack belonging to a Star is subjected simultaneously to all three modes of crack propagation, which originates from the multi-axial stress field developed under the moving contact pressure. Recall, that the opening mode $\bar{K}_{I}$ is activated by the normal tensile stress perpendicular to the crack faces, while the shearing modes $\bar{K}_{I I}$ and $\bar{K}_{I I I}$ are active due to the shear stresses resolved on the crack. If the angle $\varphi$ and the distance $b_{o f f}$ are zero (see Figs. 3), then only one half of the Star (primary crack) experiences loading and fatigue propagation, while the secondary crack is free of any mode: $\bar{K}_{I}=\bar{K}_{I I}=\bar{K}_{I I I}=0$. This is because the latter crack lays on the plane of symmetry (XZ, $y=0)$, and the shear stresses resolved on this plane $\left(\tau_{y x}\right.$ and $\tau_{y z}$ ) vanish due to the symmetry considerations, meaning that $\bar{K}_{I I}=\bar{K}_{I I I}=0$ along the entire front of this crack. The normal stress (perpendicular to the plane of symmetry) is compressive, which excludes development of $\bar{K}_{I}$ in the secondary crack (see Fig. 11). The shapes of the $\bar{K}_{I}, \bar{K}_{I I}$ and $\bar{K}_{I I I}$ distributions, corresponding to the primary crack (see Figs. 9 and 10) can be explained in terms of subsurface stresses, typical for the Hertzian point contact. When the contact zone approaches the Star and the distance $\chi$ gets close to the contact zone size, $b$, (see Fig. 11) the primary crack experiences tension, developed at the periphery of the Hertzian point contact (Ref. [27]). This is the radial stress $\sigma_{r r}$, surrounding the contact zone, as presented in Figs. 11, 12 and 13 . This stress obtains the maximum intensity at the surface $(z=0)$ and rapidly decays as the depth, $z$, increases. Therefore, only a small portion of the primary crack front is subjected to the $\bar{K}_{I}$ mode: as shown in Fig. 10, this corresponds to the crack front fraction located close to the surface, or in other words, to the angle $\theta$ close either to $0^{\circ}$ or $180^{\circ}$. On the other hand, the fraction located at higher depth 
is subjected to compressive stress (which is predominant in contact) causing the mode $\bar{K}_{I}$ to vanish, as schematically presented in Fig. 11. At the same instance ( $\chi$ is close to $b$ ), the primary crack is also subjected to the shear stress, $\tau_{x z}$, which gets the maximum intensity below the edge of the contact zone (Ref. [28]). In bearing applications, this stress is termed as the orthogonal shear stress, and is frequently considered as the main driving mechanism of RCF. In the case of elliptical bearing contact, the maximum orthogonal shear stress is located at the depth which is close to $0.5 b$, while in the current case (circular Hertzian contact) it is located at the shallower depth, which equals to $0.35 b$ (see Ref. [28]), as presented in Fig. 13. The orthogonal shear stress, $\tau_{x z}$, splits into two components along the crack front: the component normal to the crack front activates modes II and the component that is tangent to the crack front activates mode III. At the bottom of the primary crack $\left(\theta=90^{\circ}\right)$ the shear stress, $\tau_{x z}$, entirely contributes to the mode II ( $\bar{K}_{I I}$ is maximum at this point), which explains why the mode $\bar{K}_{I I I}$ vanishes at the crack bottom (see Fig. 10). At low subsurface depth $(z<<0.35 b)$, the stress $\tau_{x z}$ is negligibly low, which means that the relatively high $\bar{K}_{I I}$ values obtained on the surface (at $\theta=0^{\circ}$ and $\theta=180^{\circ}$, see Fig. 10), cannot be addressed to this stress, but to another one, which is the component $\tau_{x y}$ of the stress tensor (see Fig. 11). In bearing applications this stress is typically not considered, because it is low in the case of elliptical contact, and vanishes completely, as the contact zone gets linear (plane-stress or plane strain conditions). However, in the case of the Hertzian point contact this stress component is not negligible: it gets the maximum intensity on the surface $(z=0)$ at certain distance from the symmetry plane $(y / b \approx \pm 0.75)$ as is presented in Fig. 13. Note, that due to the symmetry considerations this stress component equals to zero at $y=0$ (on the plane of symmetry). Similarly to the tensile stress, $\sigma_{r r}$, the shear stress $\tau_{x y}$ is localized close to the surface and diminishes as the subsurface depth increases. Therefore, this stress mainly contributes to the mode $\bar{K}_{I I}$ along the crack front portion located very close to the surface. In other words, the rapid decay of the stress $\tau_{x y}$ in terms of the depth explains the existence of the two local minima on the $\bar{K}_{I I}$ curve (at $\theta \approx \pm 25^{\circ}$ ), presented in Fig. 10 .

\subsection{Crack propagation under $R C F$}

The results of the current numerical analysis (see Figs. 9 , 10) lead to some considerations regarding the RCF failure mechanism in the presence of a Star feature. The surface tensile stress inherent to the Hertzian point contact, activates the opening mode $\bar{K}_{I}$, which can be quite critical to such non-ductile material as $\mathrm{Si}_{3} \mathrm{~N}_{4}$. This mode gets maximum on the surface and can be responsible, therefore, for the crack propagation on the surface, which can be visualized without destruction of the RCF tested specimen. As was experimentally found in Ref. [12], the crack oriented perpendicularly to the rolling direction (primary crack, according to the current definition) experienced fatigue propagation, while very subtle or no propagation symptoms were observed to the sec-

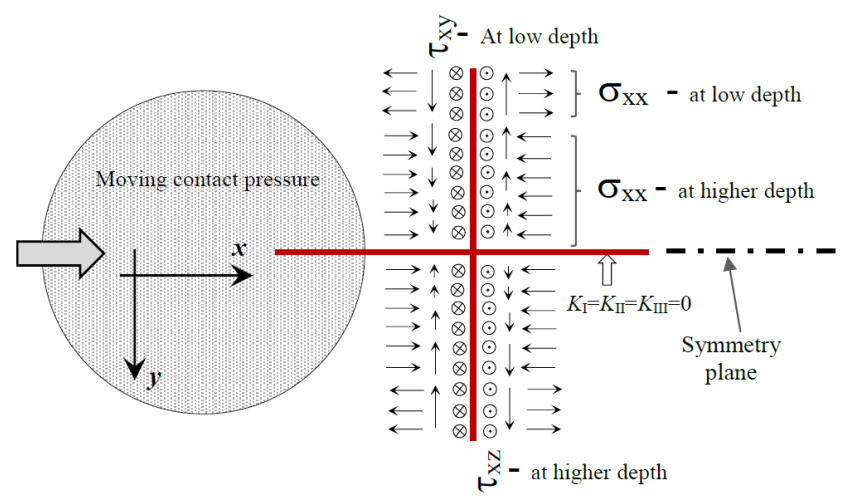

Figure 11: Star subjected to the stress field developed under the Hertzian contact.

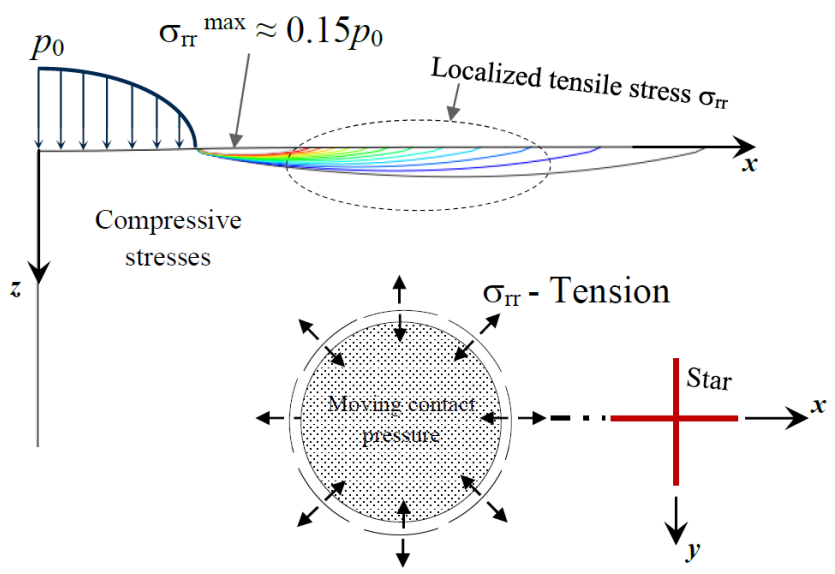

Figure 12: Localised tensile stress developed in the Hertzian contact of circular shape (point contact).

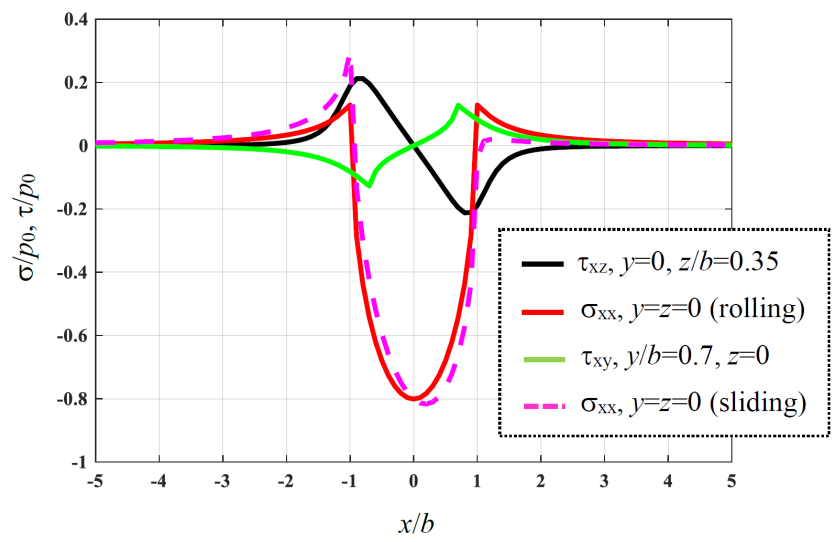

Figure 13: Distribution of main stresses driving the multiaxial crack propagation under the modes $\bar{K}_{I}, \bar{K}_{I I}$ and $\bar{K}_{I I I}$.

ond half of the Star - the crack oriented parallel to the rolling direction (secondary crack). This is consistent with the current FE analysis, stating that only one crack belonging to a Star (primary crack) is subjected to fatigue propagation, and schematically it is demonstrated in Fig. 14(a). Note that the crack propagation scenario, depicted in Fig. 14(a), is conditioned by the mixed mode, because, as is shown in Fig. 10, all three modes of crack propagation are active on the surface 

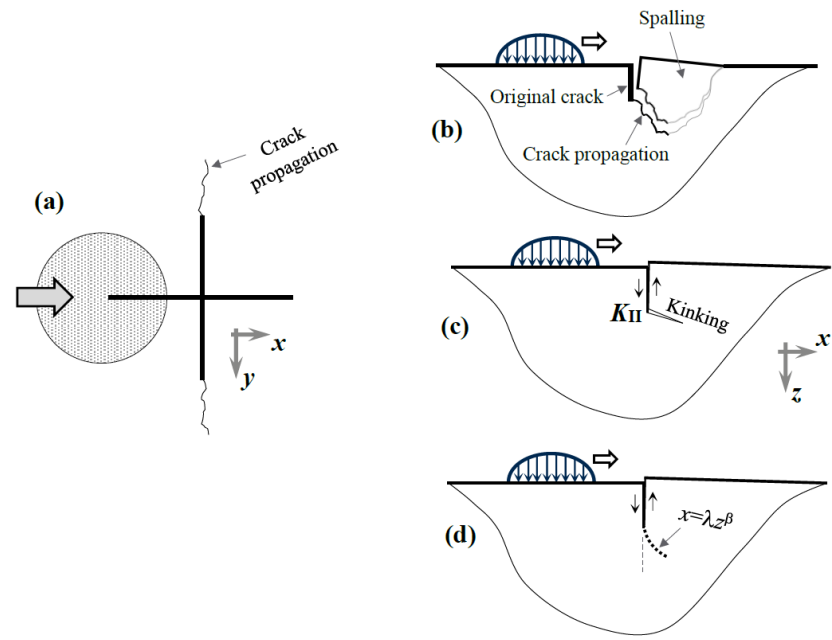

Figure 14: Schematic of the RCF failure mechanism in the presence of Star feature. Crack propagation on the surface (a), subsurface crack propagation leading to the spall formation (b), kinking failure (c) and crack propagation curve proposed by Ref. [29] (d).

(when the angle $\theta$ is either close to $0^{\circ}$ or $180^{\circ}$ ). However, in the case of limited ductility of ceramics, the $\bar{K}_{I}$ mode probably plays the dominant role in this propagation.

The crack propagation, schematically presented in Fig. 14(a), can hardly explain the formation of spalling failure (see Fig. 14(b)), because the mode $\bar{K}_{I}$ (probably driving this propagation) is restricted to a very low depth. On the other hand, the primary crack is subjected to high loading by the mode $\bar{K}_{I I}$ at the bottom $\left(\theta=90^{\circ}\right)$, which according to Fig. 10 , is the crack front point experiencing the highest loading level. The experimental observations of partially propagated Stars, obtained by the specimen cross-sectioning (see Ref. [8]), indicate that the crack propagation at the bottom can significantly deviate from its major path. This is not a new observation: it is well-known that in non-ductile materials (e.g. ceramics) crack propagation is mainly driven by local tensile stress developed at the crack tip. If a crack is loaded by mode II, then there will be maximum tension on the plane which is inclined relative to the main crack path (see e.g. Ref. [30]). Note that in the case of pure $\bar{K}_{I}$ mode, the situation is different: the crack is supposed to propagate along its original path direction. Under pure mode $\bar{K}_{I I}$ the crack kinking angle is around $70^{\circ}$ (see schematic in Fig. 14(c)), however in the case of rolling contact the situation is more complicated, because the crack is subjected to compressive normal stress (Ref. [29]) which reduces $\bar{K}_{I I}$ by activating the crack faces friction and suppressing kinking. According to Ref. [29] in such multi-axial conditions the crack is supposed to propagate along the direction, defined by the curve $x=\lambda z^{\beta}$ (see Fig. 14(d)). As the crack kinks at the bottom, its loading state can dramatically change. During a certain stage it can propagate below the surface and subsequently branch upwards, which eventually leads to the formation of spalling particle (see again Fig. 14(b)). In addition to the direction of crack propagation, Fracture Mechanics is able to predict whether a Star is supposed to propagate or is arrested. By comparing SIFs (in the case of the RCF mixed mode propagation an equivalent SIFs should be defined [31]) to the material property, termed as the fatigue crack threshold, $\Delta K_{t h}$, (for $\mathrm{Si}_{3} \mathrm{~N}_{4}$ the values of $\Delta K_{t h}$ can be found in $[9,32]$ and [33]), such a condition can be identified. Using the Paris law, the rate of fatigue crack propagation can be estimated based on SIF. Combining this law with a certain criterion predicting the crack propagation direction and using advanced computational methods (like XFEM or adaptive re-meshing), Star propagation in RCF can be simulated. However, such an advanced and computationally expensive modelling is out of the current work scope and should be addressed to a future study.

The difference observed in Fig. 10 between the first mode for two extreme contact conditions, namely rolling and sliding, is related to a difference between good and poor lubrication. So, the opening mode $\bar{K}_{I}$, in the case of sliding contact is higher than in rolling conditions along the entire crack front. This is due to the intensification of surface tension, $\sigma_{x x}$ by the friction between the ball and the substrate at the sliding contact as shown in Fig. 13 (see the solid and the dashed line, corresponding to the stress $\sigma_{x x}$ in the rolling and the sliding conditions, respectively).

\subsection{Crack face friction}

The parametric study of the current work includes the investigation of crack faces friction (defined here in terms of the Coulomb friction coefficient, $\mu_{\text {crack }}$ ) effect on SIF and it is illustrated in Fig. 15. As was mentioned before (Sec. 2), the knowledge on this phenomenon is quite limited, because the friction at crack faces can be influenced by different physical factors (e.g. crack faces roughness and their adhesive interaction, lubrication conditions), and therefore reliable experimental measurement of $\mu_{\text {crack }}$ is hardly plausible. Typically the crack faces are quite rough, which is supposed to enhance crack faces friction, but differently from subsurface cracks, Star comprises of surface crack which are not sealed from lubricant. The lubricant being entrapped into the crack can reduce friction by easing the sliding between the faces of the crack and mitigating adhesion. Regarding the latter effect, it should be mentioned, that adhesion in vacuum conditions (which is probably the case in a subsurface cracks) is supposed to be higher than in surface cracks which are exposed to air and products of lubrication (see e.g. Ref. [34]). Another effect associated with the lubricant entrapment is the crack pressurization, which can lead to dramatic increase of mode I. This effect is studied in myriads of scientific papers, however, is excluded from the current work, because the cracks of Star are perpendicular to surface and this orientation is not prone to lubricant entrapment, as was shown in Refs. [35] and [36]. Eventually, the effect of crack faces friction on the sliding modes $\bar{K}_{I I}$ and $\bar{K}_{I I I}$ (the opening mode $\bar{K}_{I}$ is not influenced by this mechanism) is presented in Fig. 15 , which apparently indicates that this friction has a beneficial effect on fatigue crack propagation: as $\mu_{\text {crack }}$ increases the $\bar{K}_{I I}$ and $\bar{K}_{I I I}$ values decrease. This is influenced by the 

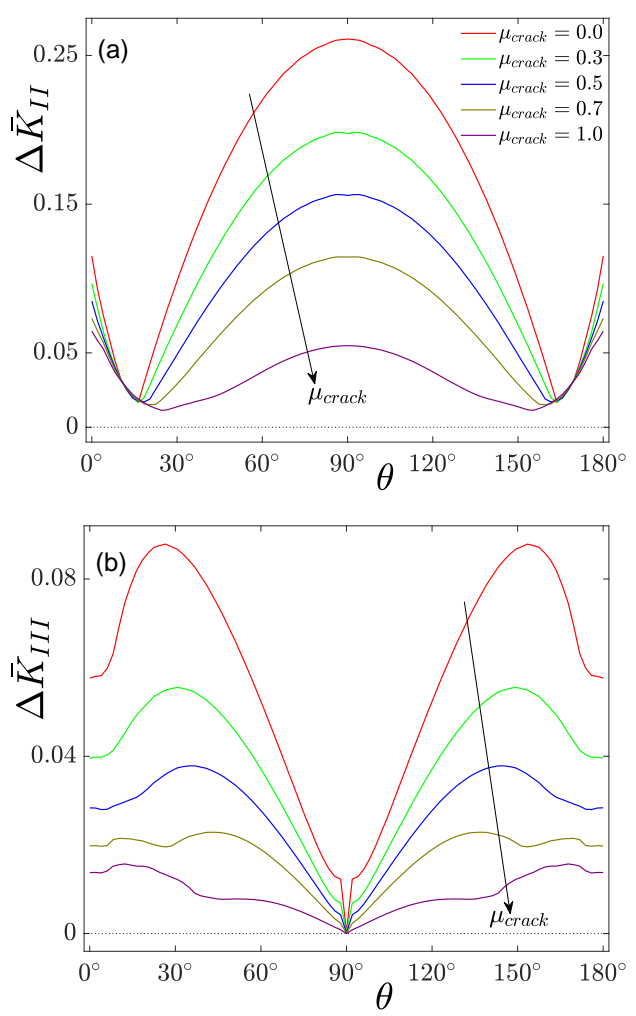

Figure 15: Distribution of (a) $\Delta \bar{K}_{I I}$ and (b) $\Delta \bar{K}_{I I I}$ along the crack front $\left(0^{\circ} \leq \theta \leq 180^{\circ}\right)$ for $\mu_{\text {crack }}=0.0,0.3,0.5,0.7,1.0$ and $C_{R} / b=0.75, d_{R} / C_{R}=0.8$.

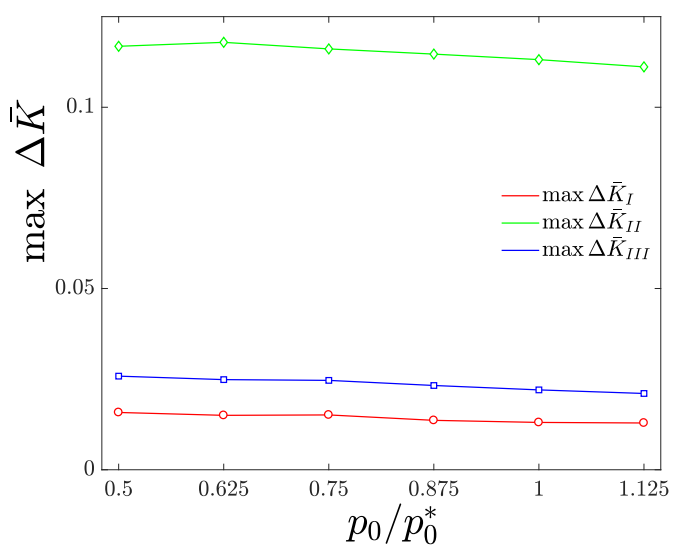

Figure 16: Dependence of maximum SIF values, $\max \Delta \bar{K}$, on the contact pressure $p_{0} / p_{0}^{*}$ for $\mu_{\text {crack }}=0.7, C_{R} / b=0.75$, $d_{R} / C_{R}=0.8$. Here $p_{0}^{*}$ stands for the constant reference maximum contact pressure and $p_{0}$ is the actual applied maximum contact pressure.

suppression of the mutual crack face sliding and associated frictional interaction.

\subsection{Effect of contact pressure}

Figure 16 shows the results of rolling contact simulations for different values of the maximum contact pressure in terms of the ratio $p_{0} / p_{0}^{*}$, where $p_{0}$ is the actual applied maximum contact pressure, and $p_{0}^{*}$ is the reference maximum contact pressure used throughout the present study ex- cept for the analysis presented in Fig. 16. In order to obtain the required $p_{0}$, the contact zone size $b$ and the crack geometry were fixed. At the absence of the crack faces friction the problem is linear, meaning that $\Delta \bar{K}$ is proportional to $p_{0}$, however such a linearity is not obvious when $\mu_{\text {crack }} \neq 0$. For the simplicity of representation, the maximum (along the entire crack front - see Fig. 10) value $\Delta \bar{K}$ value is presented in Fig. 16. So, the dependence of $\max \Delta \bar{K}$ on $p_{0} / p_{0}^{*}$ for $\mu_{\text {crack }}=0.7$ is rather linear, as it is depicted in Fig. 16 . This observation has a practical value, because it allows to reduce significantly the amount of FE simulations for the estimation of crack propagation criticality due to the varying of $p_{0}$. Since the SIF curves can be scaled in terms of $p_{0}$ (even in the case of frictional interaction at the crack faces is present) this parameter can be excluded from the parametric study.

\subsection{Non-symmetrical configuration: orientation and offset of Star feature}

So far the simulation results were demonstrated for a rather simple case at which Star is oriented at $\varphi=0^{\circ}$ and is not shifted $\left(b_{o f f}=0\right)$, leading to the symmetry of the model (see Fig. 4). However, in real testing conditions a Star can be turned at a certain angle and be shifted from the symmetry plane. Therefore, the effect of these two parameters on SIF is also investigated in the current work. It is obvious that the symmetry considered in the previous simulation (leading to the loading and propagation of only one-half of the Star (primary crack) breaks, meaning that both halves of the crack are loaded. The effect of Star orientation on the modes $\bar{K}_{I}, \bar{K}_{I I}$ and $\bar{K}_{I I I}$ is demonstrated in Figs. 17, and as is indicated by this figure the highest $\bar{K}_{I I}$ and $\bar{K}_{I I I}$ modes relate to the originally considered Star orientation, $\varphi=0^{\circ}$. On the other hand, the highest $\bar{K}_{I}$ value corresponds to the Star orientation which is close to $\varphi=30^{\circ}$. Considering the sliding modes $\bar{K}_{I I}$ and $\bar{K}_{I I I}$, the most critical Star orientation is at $\varphi=0^{\circ}$, because at this case the primary crack being oriented perpendicularly to the rolling direction, lays on the plane experiencing the highest shear stress (while the secondary crack is not loaded). However, as the angle $\varphi$ changes from zero, this shear stress splits between the primary and the secondary cracks, and when $\varphi=45^{\circ}$ the modes $\bar{K}_{I I}$ and $\bar{K}_{I I I}$ are partitioned equally between these two halves of the Star. The situation with the opening mode $\bar{K}_{I}$ is more complicated: the critical Star orientation for this mode is dependent on the Star dimensions and on the size of the contact zone, $b$. Schematically it is presented in Fig. 18, depicting the contact zone approaching to the Star. Apparently, the most critical $\varphi$ angle is the one at which the crack plane is tangent to the circular contact zone, because in this case the tensile stress $\sigma_{r r}$ being perpendicular to the crack plane entirely contributes to the mode $\bar{K}_{I}$ (see Fig. 16b). Again, depending on the dimensions of the Star and the contact zone, the most favorable (for $\bar{K}_{I}$ ) $\varphi$ angle can obtain different values. Finally, it is interesting to note that the curves in Figs. 17, indicate the existence of the discontinuity point at $\theta=90^{\circ}$. This is the point at which the two cracks of the Star intersect 

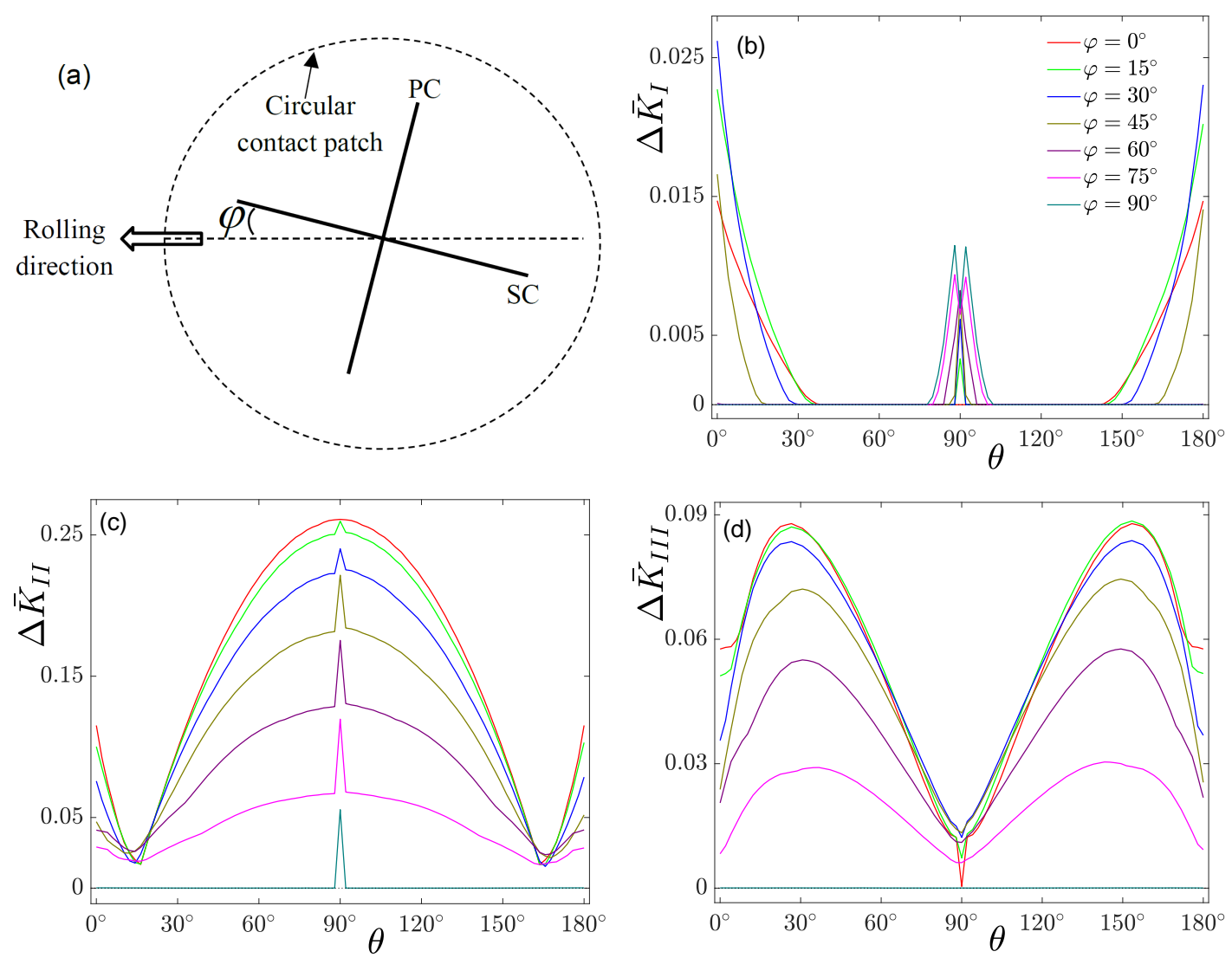

Figure 17: Schematic of the crack orientation relative to the motion of contact zone (a) and distribution of $\Delta \bar{K}$ (b), (c) and (d) along the crack front $\left(0^{\circ} \leq \theta \leq 180^{\circ}\right)$ for $\varphi=0^{\circ}, 15^{\circ}, 30^{\circ}, 45^{\circ}, 60^{\circ}, 75^{\circ}, 90^{\circ}$ and $\mu_{\text {crack }}=0, C_{R} / b=0.75, d_{R} / C_{R}=0.8$. The primary crack is perpendicular to the rolling direction for the angle $\varphi=0^{\circ}$, and parallel for $\varphi=90^{\circ}$.

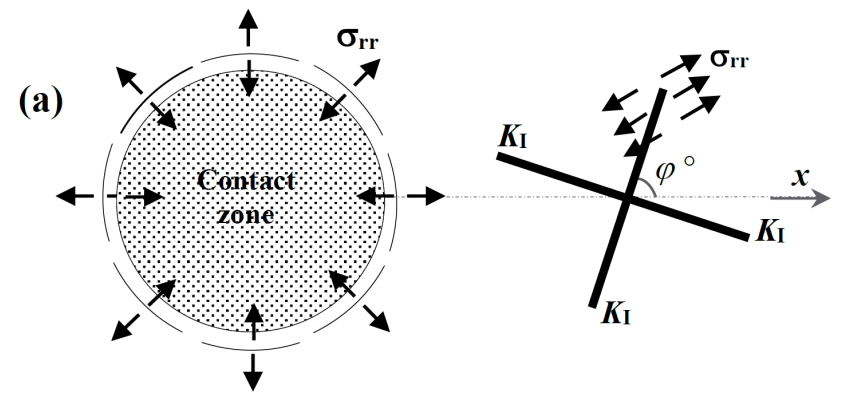

(b)

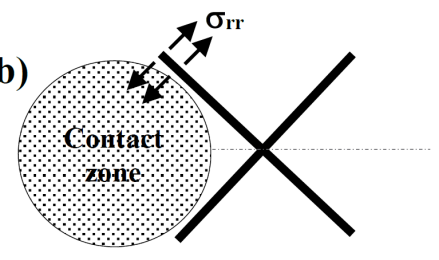

Figure 18: Development of the mode $\bar{K}_{I}$ due to the tensile radial stress, $\sigma_{r r}$, at the circumference of the contact zone (a). The $\bar{K}_{I}$ mode gets the highest value when $\sigma_{r r}$ is perpendicular to the crack (b).

each other, and the singularity at this point cannot be treated by fracture mechanics. At $\varphi=0^{\circ}$ the existence of the secondary crack can be neglected and therefore this singularity is not observed (on the previous graphs), while for $\varphi \neq 0^{\circ}$ it is not the case, because both cracks are loaded.

Another parameter leading to the breaking symmetry of the problem is $b_{o f f}$, and its effect on SIF is demonstrated in Figs. 19. The results presented in these figures correspond to the primary crack, and as expected: the distributions of $\bar{K}_{I}, K_{I I}$ and $\bar{K}_{I I I}$ are not symmetrical with respect to $\theta=90^{\circ}$ (plane of symmetry). The part of the primary crack located closer to the contact zone experiences higher SIF values, which in the case of the surface crack propagation (see Fig. 14) can be visible due to non-equal fatigue growth on the surface, being predominantly driven by $\bar{K}_{I}$.

\subsection{Parametric study on the geometry of Star feature}

Finally, the effect of Star geometry on its fatigue propagation is studied. To simplify this study the parameters $\varphi$ and $b_{o f f}$ are switched to zero (providing the symmetry of the problem) and no friction at the crack faces is assumed. Fig. 20(a) depicts the dependence of $\max \Delta \bar{K}$ on the dimensionless radial crack depth $d_{R} / C_{R}$ in rolling and sliding contact. The dimensionless radial crack length is constant $C_{R} / b=0.75$ and frictionless crack face assumed. The solid curves represent rolling, the dashed curves - sliding with $\mu_{\text {surface }}=0.05$ (similar to rolling) and dash-dotted curves with $\mu_{\text {surface }}=0.15$. As the dimensionless radial depth increases, the maximum values of the second mode slightly 

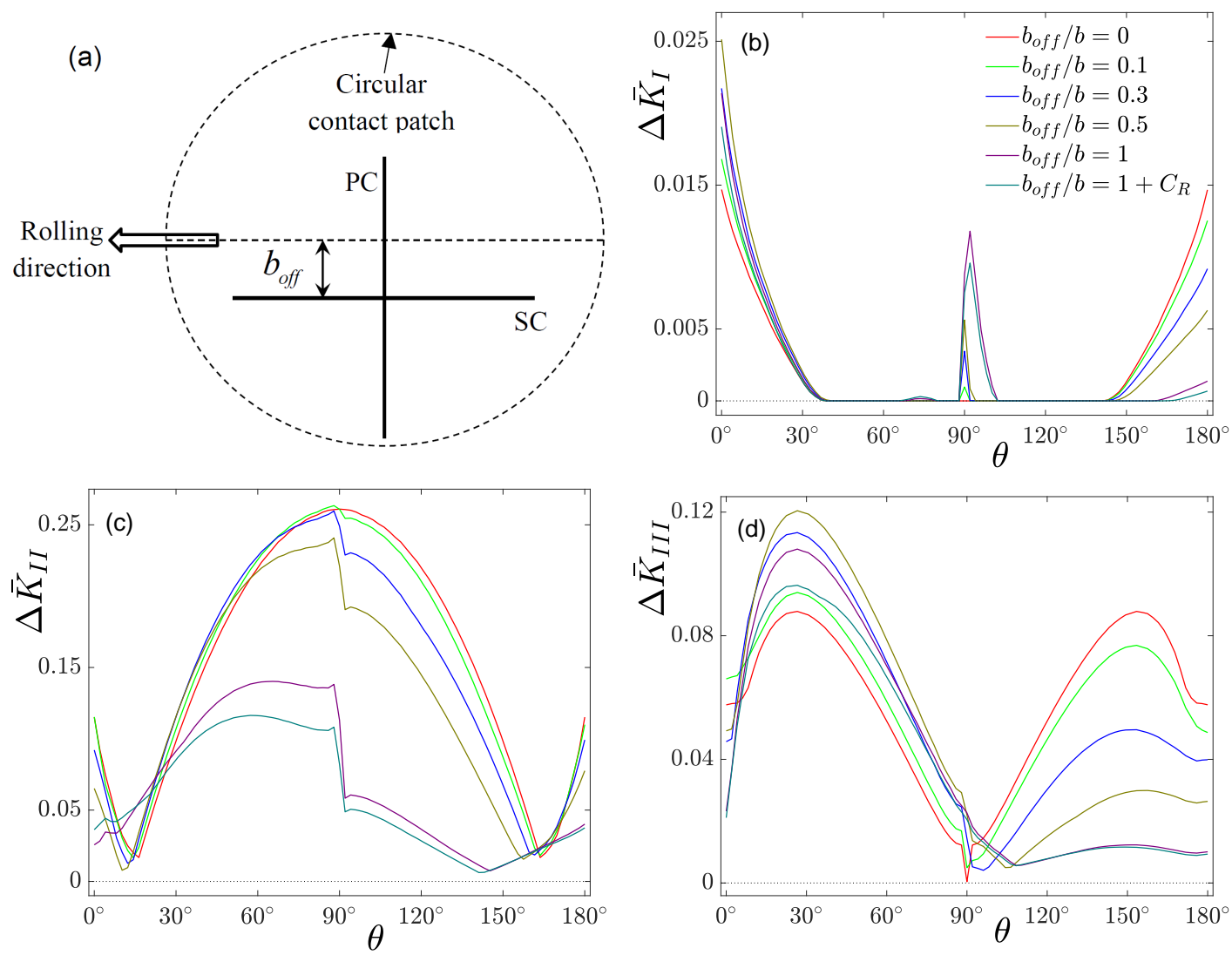

Figure 19: Schematic of the offset distance between the intersection of the cracks forming the Star and the rolling path (a) and distribution of $\Delta \bar{K}$ (b), (c) and (d) along the crack front $\left(0^{\circ} \leq \theta \leq 180^{\circ}\right)$ for $b_{o f f} / b=0,0.1,0.3,0.5,1,1+C_{R}$ with $\varphi=0^{\circ}$, $\mu_{\text {crack }}=0, C_{R} / b=0.75, d_{R} / C_{R}=0.8$.

decrease, and the maximum values of the third mode slightly increase, whereas the third mode is almost constant. Such a behaviour demonstrates moderate dependence of SIF on the depth of the Star feature. However, as the SIFs are represented in dimensionless form, i.e. mode II was normalized by $\sqrt{d_{R}}$, the possibility of the spall formation (shown in Fig. 14(b)) may also depend on the radial depth. The sliding in Fig. 20(a) affects mainly the first mode, with a minor affect on the second mode for shallow depth. When the friction coefficient at the surface increases from 0.05 to 0.15 and sliding is considered, only the first mode increases, with an exception for $C_{R} / b=1$. The dependence of $\max \Delta \bar{K}$ on the dimensionless radial crack length $C_{R} / b$ for constant dimensionless depth $d_{R} / C_{R}=0.8$ and $\mu_{\text {crack }}=0$ is shown in Fig. 20(b). The radial crack length $C_{R}$ is constant, so the contact radius $b$ increases with an increase in the ball radius $R_{w}$. The non-monotonic behaviour of the second and the third modes is related to the non-monotonic development of the shear stresses as the contact radius grows. The effect of sliding on the first mode decreases with a decrease in the contact radius, so that for $C_{R} / b=2$ there is almost no difference in rolling and sliding for any $\mu_{\text {surface }}$ considered for the simulation parameters.

\section{Concluding remarks}

The rolling and sliding contact problem in presence of surface Star features was parametrically investigated with Finite Element Method. According to the study, crack propagation of ceramics at the surface may be explained by the tensile stress and the dominant first SIF mode. Shear stress and the second SIF mode, however, is the main driving mechanism behind possible crack propagation at the depth of the material, that may lead to failures such as spalling. It was found that the first SIF mode is higher in case of sliding than in rolling contact, while the other modes are almost unaffected by the contact conditions, as expected. For an increase of the crack face friction a corresponding decrease in the second and the third SIF modes was obtained, with the first mode remained unchanged for any crack face friction. The latter friction, as it appears from the study, does not affect the dependence of SIFs on maximum contact pressure: it is linear regardless the friction at the crack face. Non-symmetrical orientation of the Star feature leads to decrease in the second and the third modes, following a split of the shear stress between these modes. $\bar{K}_{I}$, however, it significantly increases for the orientation $\varphi=30^{\circ}$, as the crack plane is tangent to the contact zone. An increase in the SIFs was found for a primary crack that is close to the contact zone, with a non-zero shift of the Star feature from 

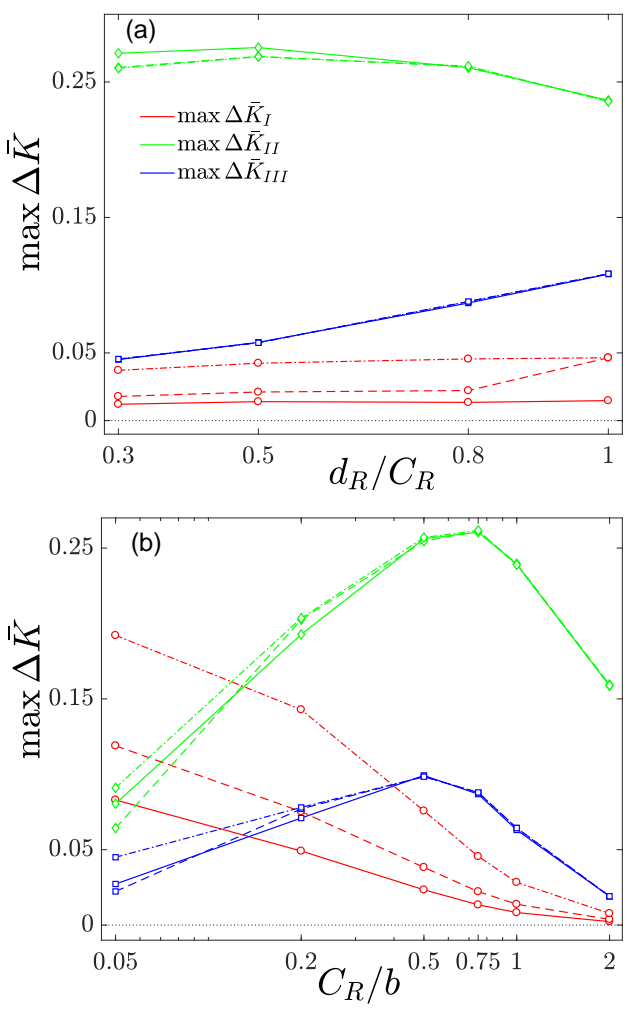

Figure 20: Dependence of $\max \Delta \bar{K}$ in rolling and sliding contact on (a) the dimensionless radial crack depth $d_{R} / C_{R}$ for $C_{R} / b=0.75$ : the solid curve represents rolling, the dashed curve - sliding with $\mu_{\text {surface }}=0.05$ (similar to rolling) and dashdotted curve with $\mu_{\text {surface }}=0.15$; (b) dependence of $\max \Delta \bar{K}$ on the dimensionless radial crack length $C_{R} / b$ for $d_{R} / C_{R}=0.8$. Frictionless crack face $\left(\mu_{\text {crack }}=0\right)$ is assumed.

a rolling or sliding direction. It was also observed that the SIFs slightly depend on the depth of the Star feature, but show non-monotonic behaviour of shearing modes for a decrease of the contact zone.

\section{Acknowledgments}

This research was supported by SKF Research \& Technology Development.

\section{References}

[1] L. Wang, R. Snidle, L. Gu, Rolling contact silicon nitride bearing technology: a review of recent research, Wear 246 (1) (2000) 159 173. doi:https://doi.org/10.1016/S0043-1648(00)00504-4.

URL http://www.sciencedirect.com/science/article/pii/ S0043164800005044

[2] C. Vieillard, Y. Kadin, G. Morales-Espejel, A. Gabelli, An experimental and theoretical study of surface rolling contact fatigue damage progression in hybrid bearings with artificial dents, Wear 364-365 (2016) 211 - 223. doi :https://doi.org/10.1016/j. wear. 2016.07.016. URL http://www.sciencedirect.com/science/article/pii/ S0043164816301582

[3] K. Niihara, R. Morena, D. P. H. Hasselman, Evaluation of kic of brittle solids by the indentation method with low crack-to-indent ratios, Journal of Materials Science Letters 1 (1) (1982) 13-16. doi: 10.1007/BF00724706.

URL https://doi.org/10.1007/BF00724706
[4] Y. Kadin, M. Mazaheri, V. Zolotarevskiy, C. Vieillard, M. Hadfield, Finite elements based approaches for the modelling of radial crack formation upon vickers indentation in silicon nitride ceramics, Journal of the European Ceramic Society 39 (14) (2019) 4011 4022. doi : https://doi.org/10.1016/j. jeurceramsoc. 2019.05.058.

URL http://www. sciencedirect.com/science/article/pii/ S0955221919303772

[5] M. Hadfield, T. A. Stolarski, R. T. Cundill, Failure modes of ceramics in rolling contact, Proceedings: Mathematical and Physical Sciences 443 (1919) (1993) 607-621.

URL http://www. jstor.org/stable/52422

[6] M. Hadfield, S. Tobe, T. Stolarski, Subsurface crack investigation on delaminated ceramic elements, Tribology International 27 (5) (1994) 359 - 367. doi: https://doi .org/10.1016/0301-679x(94)90030-2.

URL http://www.sciencedirect.com/science/article/pii/ 0301679X94900302

[7] K. Kida, T. Urakami, T. Yamazaki, K. Kitamura, Surface crack growth of silicon nitride bearings under rolling contact fatigue, Fatigue \& Fracture of Engineering Materials \& Structures 27 (8) (2004) 657-668. doi:10.1111/j.1460-2695.2004.00786.x.

URL https://onlinelibrary.wiley.com/doi/abs/10.1111/j. 1460-2695.2004.00786.x

[8] K. Kida, M. Saito, K. Kitamura, Flaking failure originating from a single surface crack in silicon nitride under rolling contact fatigue, Fatigue \& Fracture of Engineering Materials \& Structures 28 (12) (2005) 1087-1097. doi:10.1111/j.1460-2695.2005.00947.x.

URL https://onlinelibrary.wiley.com/doi/abs/10.1111/j. 1460-2695.2005.00947.x

[9] K. Kida, T. Honda, E. C. Santos, Mode ii surface crack growth under rolling contact fatigue and cyclic shear stress in si3n4, WIT Transactions on Engineering Sciences 76 (2012) 175 - 187. doi:https://doi .org/10.2495/TD120151.

URL https://www.witpress.com/elibrary/ wit-transactions-on-engineering-sciences/76/23771

[10] Y. Kadin, A. Rychahivskyy, Modeling of surface cracks in rolling contact, Materials Science and Engineering: A 541 (2012) 143 151. doi:https://doi.org/10.1016/j.msea.2012.02.016.

URL http://www.sciencedirect.com/science/article/pii/ S0921509312002067

[11] J. Lai, Y. Kadin, C. Vieillard, Characterization and modelling of the degradation of silicon nitride balls with surface missing-material defects under lubricated rolling contact conditions, Wear 398-399 (2018) 146 - 157. doi:https://doi.org/10.1016/j. wear. 2017.12.007. URL http://www.sciencedirect.com/science/article/pii/ S0043164817313091

[12] R. J. Hanzal, Rolling contact fatigue failures in silicon nitride and their detection, Ph.D. thesis, University of Southampton (September 2013).

URL https://eprints. soton.ac.uk/361698/

[13] Y. Kadin, S. Strobl, C. Vieillard, P. Wijnbergen, V. Ocelik, In-situ observation of crack propagation in silicon nitride ceramics, Procedia Structural Integrity 7 (2017) 307 - 314, 3rd International Symposium on Fatigue Design and Material Defects, FDMD 2017. doi:https://doi.org/10.1016/j.prostr. 2017.11.093.

URL http://www.sciencedirect.com/science/article/pii/ S2452321617304493

[14] S. T. Mousavi, N. Richart, C. Wolff, J. Molinari, Dynamic crack propagation in a heterogeneous ceramic microstructure, insights from a cohesive model, Acta Materialia 88 (2015) 136 - 146. doi:https://doi .org/10.1016/j. actamat.2015.01.003.

URL http://www.sciencedirect.com/science/article/pii/ S1359645415000075

[15] Q. Fan, C. Chai, Q. Wei, P. Zhou, Y. Yang, Elastic anisotropy and electronic properties of si3n4 under pressures, AIP Advances 6 (8) (2016) 085207. arXiv:https://doi.org/10.1063/1.4961117, doi:10. 1063/1.4961117.

URL https://doi.org/10.1063/1.4961117

[16] T. Anderson, Fracture Mechanics: Fundamentals and Applications, 
4th Edition, CRC Press, 2017.

URL https://www.crcpress.com/Fracture-Mechanics-Fundamenta\% ls-and-Applications-Fourth-Edition/Anderson/p/book/9781498728133

[17] B. Trollé, M.-C. Baietto, A. Gravouil, S. Mai, T. Nguyen-Tajan, Xfem crack propagation under rolling contact fatigue, Procedia Engineering 66 (2013) 775 - 782, fatigue Design 2013, International Conference Proceedings. doi:https://doi.org/10.1016/j.proeng. 2013.12.131. URL http://www.sciencedirect.com/science/article/pii/ S1877705813019644

[18] P. Pyrzanowski, Application of experimental results to numerical models of fatigue cracks propagating in the rolling contact zone, Journal of Theoretical and Applied Mechanics 44 (3).

URL http://www.ptmts.org.pl/jtam/index.php/jtam/article/vi\%ew/ v44n3p731

[19] A. A. Wereszczak, W. Wang, Y. Wang, M. Hadfield, W. Kanematsu, T. P. Kirkland, O. M. Jadaan, Rolling contact fatigue of ceramics, doi: 10.2172/947572.

[20] D. Dowson, Thin films in tribology, in: D. Dowson, C. Taylor, T. Childs, M. Godet, G. Dalmaz (Eds.), Thin Films in Tribology, Vol. 25 of Tribology Series, Elsevier, 1993, pp. $3-12$. doi:https://doi.org/10.1016/S0167-8922(08)70358-4.

URL http://www.sciencedirect.com/science/article/pii/ S0167892208703584

[21] K. L. Johnson, Contact Mechanics, Cambridge University Press, 1985. doi:10.1017/Св09781139171731.

[22] A. W. Awan, Defect tolerance assessment of silicon nitride in rolling contact, Ph.D. thesis, Bournemouth University (July 2015).

URL http://eprints. bournemouth. ac.uk/25044/

[23] S. Suresh, Fatigue of Materials, 2nd Edition, Cambridge University Press, 1998. doi:10.1017/СB09780511806575.

[24] S. Strobl, T. Lube, P. Supancic, O. Schoppl, R. Danzer, Surface strength of balls made of five structural ceramic materials evaluated with the notched ball test (nbt), Journal of the European Ceramic Society 37 (15) (2017) 5065 - 5070. doi:https://doi.org/10.1016/j. jeurceramsoc. 2017.06.045. URL http://www.sciencedirect.com/science/article/pii/ S0955221917304685

[25] H. Tada, P. C. Paris, G. R. Irwin, The Stress Analysis of Cracks Handbook, Third Edition, ASME Press, 2000. doi :10.1115/1.801535. URL https://doi.org/10.1115/1.801535

[26] S. Strobl, P. Supancic, T. Lube, R. Danzer, Surface crack in tension or in bending - a reassessment of the newman and raju formula in respect to fracture toughness measurements in brittle materials, Journal of the European Ceramic Society 32 (8) (2012) 1491 - 1501. doi:https://doi.org/10.1016/j. jeurceramsoc. 2012.01.011. URL http://www.sciencedirect.com/science/article/pii/ S0955221912000258

[27] A. C. Fischer-Cripps, Introduction to Contact Mechanics, 2nd Edition, Springer US, 2007. doi :10.1007/978-0-387-68188-7.

[28] T. A. Harris, M. N. Kotzalas, Rolling Bearing Analysis, 5th Edition, CRC Press, 2006.

URL https://www.crcpress.com/Rolling-Bearing-Analysis---2-\% Volume-Set/Harris-Kotzalas/p/book/9780849381676

[29] P. Isaksson, On crack growth under compressive stresses, Ph.D. thesis, Luleå University of Technology (2001).

URL http://hdl. handle. net/2043/8609

[30] G. Hua, M. W. Brown, K. J. Miller, Mixed-mode fatigue thresholds, Fatigue \& Fracture of Engineering Materials \& Structures 5 (1) (1982) 1-17. doi:10.1111/j.1460-2695.1982.tb01220.x

URL https://onlinelibrary.wiley.com/doi/abs/10.1111/j. 1460-2695.1982. tb01220.x

[31] J. Qian, A. Fatemi, Mixed mode fatigue crack growth: A literature survey, Engineering Fracture Mechanics 55 (6) (1996) 969 - 990. doi:https://doi.org/10.1016/S0013-7944(96)00071-9.

URL http://www.sciencedirect.com/science/article/pii/ S0013794496000719

[32] S. G. Warrier, D. C. Jarmon, H. A. Chin, Finite Element Analysis of the Critical Flaw Size in Hybrid Silicon Nitride Bearing Ball Vol- ume 4: Manufacturing Materials and Metallurgy; Ceramics; Structures and Dynamics; Controls, Diagnostics and Instrumentation; Education. doi:https://doi.org/10.1115/2000-GT-0065.

[33] R. O. Ritchie, R. H. Dauskardt, Cyclic fatigue of ceramics: a fracture mechanics approach to subcritical crack growth and life prediction, Journal of the Ceramic Society of Japan 99:1154.

[34] A. Iwabuchi, T. Kayaba, K. Kato, Effect of atmospheric pressure on friction and wear of $0.45 \%$ c steel in fretting, Wear 91 (3) (1983) 289 -305. doi:https://doi.org/10.1016/0043-1648(83)90074-1.

URL http://www.sciencedirect.com/science/article/pii/ 0043164883900741

[35] Y. Murakami, C. Sakae, K. Ichimaru, T. Morita, Experimental and Fracture Mechanics Study of the Pit Formation Mechanism Under Repeated Lubricated Rolling-Sliding Contact: Effects of Reversal of Rotation and Change of the Driving Roller, Journal of Tribology 119 (4) (1997) 788-796. doi:10.1115/1.2833886.

URL https://doi.org/10.1115/1.2833886

[36] D. Ne'lias, M.-L. Dumont, F. Couhier, G. Dudragne, L. Flamand, Experimental and Theoretical Investigation on Rolling Contact Fatigue of 52100 and M50 Steels Under EHL or Micro-EHL Conditions, Journal of Tribology 120 (2) (1998) 184-190. doi:10.1115/1.2834407. URL https://doi.org/10.1115/1.2834407 